\title{
ẢNH HƯỞNG CỦA NGUỒN LỰC DOANH NGHIỆP ĐẾN HIỆ QUẢ HOẠT ĐỘNG KINH DOANH CỦA DOANH NGHIỆP NHỎ VÀ VÙA TẠI VIẸT NAM
}

\author{
VÕ VĂN DÚT, TRÀN QUẾ ANH, PHẠM BÍCH NGỌC \\ Truờng Đại học Cần Tho; \\ vvdut@ctu.edu.vn,tqanh@ctu.edu.vn,ngocb1302486@student.ctu.edu.vn
}

Tóm tắt. Bài viết này nghiên cứu tác động điều tiết của mức độ thực hiện hợp đồng phụ công nghiệp đến mối quan hệ giữa nguồn lực bên trong doanh nghiệp nhỏ và vừa (DNNVV) và hiệu quả hoạt động kinh doanh (HQHĐKD) tại Việt Nam. Sử dụng Lý thuyết nguồn lực công ty, nghiên cứu này giả thuyết rằng, doanh nghiệp càng tăng cường thực hiện hợp đồng phụ thì vốn từ vay nợ và nguồn lực marketing càng làm giảm hiệu quả hoạt động của doanh nghiệp, trong khi đó trình độ của chủ doanh nghiệp làm tăng hiệu quả hoạt động của doanh nghiệp. Nghiên cứu sử dụng bộ dữ liệu điều tra DNNVV hoạt động trong lĩnh vực chế tạo ở Việt Nam. Kết quả kiểm định tại 260 DNNVV có thực hiện hợp đồng phụ đã chỉ ra rằng, các giả thuyết đặt ra không thể bác bỏ. Tuy nhiên, nghiên cứu thất bại để chỉ ra bằng chứng thực nghiệm đối với vai trò của hợp đồng phụ đến mối quan hệ giữa tỷ lệ nợ vay và HQHĐKD của doanh nghiệp.

Từ khoá. doanh nghiệp nhỏ và vừa, hợp đồng phụ, hiệu quả hoạt động kinh doanh, nguồn lực doanh nghiệp.

\section{THE EFFECT OF ENTERPRISE'S RESOURCES ON PERFORMANCE OF SMALL AND MEDIUM ENTERPRISE IN VIETNAM}

\begin{abstract}
The study investigates the moderating effect of subcontract on the relationship between resources and small-medium enterprise (SME)'s performance in Vietnam. Building upon resource-based view, the hypotheses are proposed that the effect on loan and marketing resource on the performance of sub-contract SME is lesser than that of non sub-contract SME, whereas the effect of SME manager's education on its performance is higher. 260 sub-contract SMEs from enterprise survey data of the World Bank were used to test the hypotheses. Regression reveals that the empirical findings are strongly supported the proposed hypotheses. The study however fails to find the evidence on the role of subcontract on the relationship between debt and SME's performance.
\end{abstract}

Keywords. SMEs, subcontracting, performance, firm resource.

\section{GIÓI THIẸU}

Hiệu quả hoạt động của doanh nghiệp, đặc biệt là các DNNVV là chủ đề được nhiều nhà nghiên cứu quan tâm trong những thập niên qua [10], [3], [9] bởi số lượng doanh nghiệp và vai trò quan trọng của các doanh nghiệp này trong việc đóng góp vào GDP của quốc gia, v.v. Theo Tổng cục thống kê thông tin, tính đến tháng cuối năm 2016, trong số 477.808 doanh nghiệp Việt Nam có đến $97 \%$ là DNNVV; trong đó gần $60 \%$ doanh nghiệp có qui mô rất nhỏ, vốn cũng như điều kiện kỹ thuật lạc hậu dẫn đến hiệu quả kinh doanh chưa cao. Về mặt học thuật, một trong những lý thuyết để giải thích những yếu tố ảnh hưởng đến HQHĐKD của doanh nghiệp là Lý thuyết nguồn lực [18]. Theo quan điểm này, khả năng doanh nghiệp kiểm soát các nguồn lực có giá trị, hiếm, khó bắt chước và thay thế sẽ tạo ra được những lợi thế cạnh tranh bền vững cho doanh nghiệp, từ đó tăng cường HQHĐKD. Song song đó, thuê ngoài (outsourcing) ngày càng được quan tâm bởi các học giả trong và ngoài nước. Trong đó, hơp đồng phu được hiểu là việc công ty sản xuất chính có quy mô lớn và thuê những doanh nghiệp nhỏ hơn thực hiện sản xuất các linh kiện, bộ phận phục vụ cho việc hoàn thành sản phẩm chính cuối cùng của mình theo một cam kết hợp đồng [7]. Đến nay, trên thế giới đã xuất hiện không ít tranh luận trái chiều xoay quanh tác động của các hợp đồng phụ. Theo [8], các DNNVV hạn chế về nguồn lực hơn rất nhiều so với các doanh nghiệp lớn, do vậy các DNNVV sẽ thực hiện hợp đồng phụ nên phải phụ thuộc vào bên giao hợp đồng về 
đầu vào cũng như công nghệ, dẫn đến tình trạng không thể nắm hết chuỗi giá trị sản xuất, khó đạt được hiệu quả cao trong kinh doanh. Ngược lại, một số nghiên cứu khác cho rằng $\mathrm{DNNVV}$ có thể tận dụng việc thực hiện hợp đồng phụ để khai thác các lợi ích như mở rộng mối quan hệ, học hỏi kinh nghiệm quản trị, cập nhật, chuyển giao công nghệ hiện đại, khắc phục những yếu điểm vốn có và sản xuất hiệu quả hơn [5]. Tuy nhiên, hiện nay vấn đề này vẫn còn bỏ ngỏ tại Việt Nam. Vì vậy, mục tiêu của bài viết này là nghiên cứu nguồn lực của DNNVV ảnh hưởng như thế nào đến HQHĐKD của DNNVV? Đồng thời, xem xét vai trò của việc thực hiện hợp đồng phụ đến hiệu quả sử dụng nguồn lực doanh nghiệp vào hoạt động sản xuất kinh doanh của các DNNVV tại Việt Nam. Bằng cách sử dụng Lý thuyết nguồn lực công ty, các biện luận về mặt lý thuyết của bài viết được phát triển để bổ sung những kiến thức về vai trò của hợp đồng phụ trong việc xem xét mối quan hệ giữa nguồn lực và HQHĐKD của DNNVV hiện nay. Thêm vào đó, bằng chứng thực nghiệm được kiểm định tại các DNNVV ở Việt Nam là tài liệu tham chiếu quan trọng để các nhà quản lý nhìn nhận lại vai trò của việc thực hiện hợp đồng phụ đối với nguồn lực và HQHĐKD của đơn vị mình. Từ đó, có những định hướng và chiến lược phù hợp nhằm phát triển doanh nghiệp một cách bền vững góp phần phát triển kinh tế của quốc gia.

\section{CƠ SỞ LÝ THUYẾT VÀ CÁC GIẢ THUYÊT}

Theo Lý thuyết nguồn lực [18], nguồn lực chính là cơ sở để tạo ra sự khác biệt trong HQHĐKD giữa các doanh nghiệp trong cùng ngành [1]. Thực tế, do hoạt động kinh doanh của một doanh nghiệp là một phạm trù rất rộng nên các loại nguồn lực doanh nghiệp ảnh hưởng đến hoạt động kinh doanh cũng rất đa dạng. Để phục vụ cho mục tiêu đã đề ra, bài viết này tiến hành tập trung vào các loại nguồn lực cho đối tượng các nhà thầu phụ công nghiệp. Theo các học giả nghiên cứu về nguồn lực của DNNVV, nguồn lực nhà thầu phụ công nghiệp gồm tỷ lệ nợ vay, nguồn lực marketing, trình độ của chủ doanh nghiệp [6]. Từ quan điểm của Lý thuyết nguồn lực, các giả thuyết cho mối quan hệ giữa nguồn lực và HQHĐKD của doanh nghiệp được phát triển.

\subsection{Tỷ lệ nọ̣ vay}

[6] cho rằng, ủng hộ cho mối quan hệ đồng biến của vốn vay và HQHĐKD. Lý thuyết này lập luận rằng, các doanh nghiệp có số nợ vay cao sẽ càng có nhiều động lực để sản xuất hơn. Ngoài ra, theo [14], các DNNVV thường đều thiếu vốn hoạt động, vì thế việc tiếp cận vốn vay đúng lúc sẽ góp phần đáng kể khắc phục những khó khăn trở ngại trong sản xuất. Vì những lý do trên, giả thuyết đặt ra là:

Giả thuyết (GT) 1: Tỷ lệ nợ vay càng lớn thì HQHĐKD của doanh nghiệp càng cao.

\subsection{Nguồn lực marketing}

Theo [8], khi càng tăng chi phí cho marketing, đồng nghĩa với tăng cường hoạt động quảng bá, xây dựng kênh phân phối, v.v. các doanh nghiệp sẽ có thể tăng cường thị phần và từ đó tăng thêm lợi nhuận. Vì vậy, giả thuyết thứ hai được đề nghị như sau:

Giả thuyết (GT) 2: Nguồn lực marketing có tác động đồng biến với HQHĐKD của doanh nghiệp.

\subsection{Trình độ chủ doanh nghiệp}

[14] chỉ ra rằng trình độ của chủ doanh nghiệp có tương quan thuận với HQHĐKD của DNNVV. Ngoài ra, dù có thể không phải là đối tượng lao động trực tiếp tham gia sản xuất, nhưng khi có trình độ học vấn càng cao, các chủ doanh nghiệp sẽ càng có khả năng tiếp cận các phương thức khoa học quản lý hiện đại giúp công ty phát triển hơn và có nhiều cơ hội hơn, đồng thời có mối quan hệ rộng hơn, thông hiểu về các thể chế, quy định chính sách nhiều hơn [9]. Vì những lý do trên, nghiên cứu đề xuất giả thuyết thứ 3 như sau:

Giả thuyết (GT) 3: Trình độ chủ doanh nghiệp có ảnh hưởng tích cực đến HQHĐKD của doanh nghiệp.

\subsection{Vai trò của thực hiện hợp đồng phụ}

Với các doanh nghiệp thực hiện hợp đồng thuê ngoài thì năng lực tài chính rất quan trọng trong việc hình thành và duy trì mối quan hệ kinh doanh cùng các khách hàng lớn nước ngoài, từ đó có thêm nhiều cơ hội kinh doanh quốc tế [8]. Tuy nhiên, để có lượng vốn lớn tăng cường sản xuất theo hợp đồng phụ, doanh nghiệp càng vay nợ nhiều sẽ càng gặp áp lực trả lại lớn, nhiều khó khăn và rủi ro trong thanh 
khoản. [12] cho rằng áp lực tài chính quá cao sẽ gây cản trở cho các chính sách về lao động và vốn đầu tư, hai nhân tố quan trọng tác động lên HQHĐKD. Do đó, bài viết này đề nghị giả thuyết như sau:

Giả thuyết (GT) 4a: Doanh nghiệp có thực hiện hợp đồng phụ thì tác động thuận chiều của tỷ lệ nợ vay đến $\mathrm{HQH} Đ K D$ của doanh nghiệp là yếu hơn so với các doanh nghiệp không thực hiện hợp đồng phụ.

Theo [2], từ trước đến nay các nhà thầu phụ công nghiệp vẫn trung thành với đường lối tập trung nguồn lực cốt lõi cho hoạt động sản xuất, còn các hoạt động như marketing, thiết kế và cải tiến sản phẩm vẫn chưa nhận được sự quan tâm đúng mức. Vì vậy, các nhà thầu phụ hầu như hoàn toàn phụ thuộc vào bên giao hợp đồng thực hiện các hoạt động trên, vì họ cũng chính là đối tượng sẽ tương tác với thị trường tiêu thụ cuối cùng. Bởi lẽ đó, khi muốn đẩy mạnh HQHĐKD bằng việc tăng nguồn lực marketing, những doanh nghiệp này sẽ không đủ kinh nghiệm cũng như không thể chủ động đề ra những chiến lược marketing hiệu quả. Do đó, giả thuyết kế tiếp được đề ra như sau:

Giả thuyết (GT) 4b: Doanh nghiệp có thực hiện hợp đồng phụ thì mối quan hệ đồng biến của nguồn lực marketing và $\mathrm{HQH} Đ K D$ của doanh nghiệp là yếu hơn so với các doanh nghiệp không thực hiện hợp đồng phụ.

[8] cho rằng trình độ chuyên môn của người quản lý DNNVV sẽ được tăng cường qua việc thực hiện hợp đồng phụ do qua những liên kết này, chủ các doanh nghiệp có nhiều cơ hội hơn trong tìm kiếm và mở rộng mạng lưới khách hàng, học hỏi những bài học kinh nghiệm, cách thức quản trị kinh doanh từ các đối tác lớn, từ đó áp dụng hiệu quả hơn cho chính mô hình kinh doanh của doanh nghiệp mình. Vì thế, kỳ vọng đặt ra ở giả thuyết $4 \mathrm{c}$ là:

Giả thuyết (GT) 4c: Doanh nghiệp có thực hiện hợp đồng phụ thì ảnh hưởng đồng biến của trình độ chủ doanh nghiệp đến HQHĐKD của doanh nghiệp là mạnh hơn so với doanh nghiệp không thực hiện hợp đồng phụ.

Từ các lập luận trên, mô hình nghiên cứu được tổng hợp trong Hình 1:

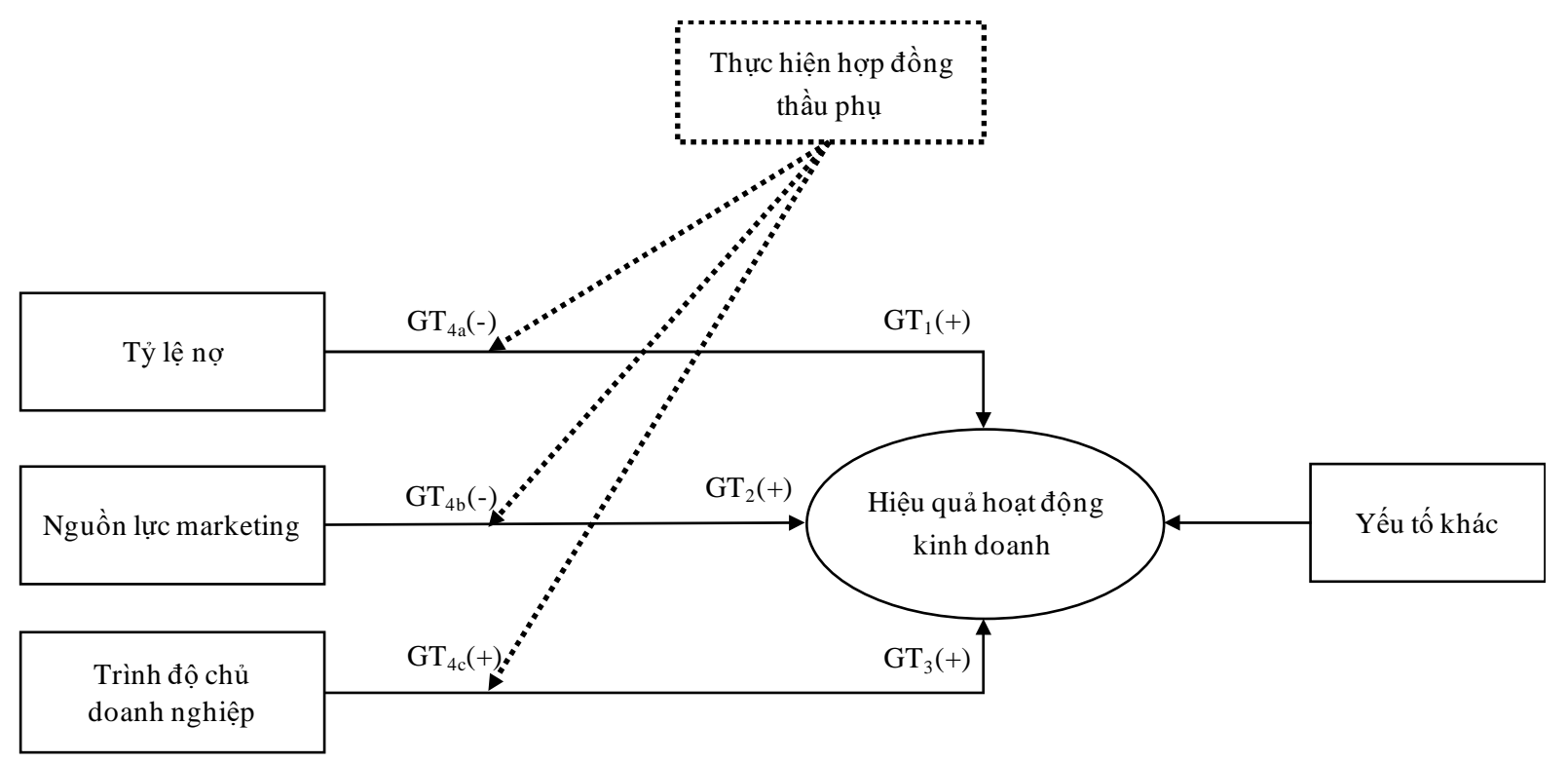

Hình 1: Mô hình nghiên cứu

\section{PHƯƠNG PHÁP NGHIÊN CÚU}

\subsection{Dữ liệu sử dụng}

Để kiểm định các giả thuyết đã nêu, nghiên cứu này sử dụng số liệu thứ cấp thu từ cuộc điều tra doanh nghiệp vào năm 2015 do Viện Nghiên cứu quản lý kinh tế Trung ương - Bộ Kế hoạch và Đầu tư, Viện Khoa học lao động và xã hội - Bộ Lao động Thương binh và Xã hội, Khoa Kinh tế - Trường Đại học tổng hợp Copenhagen, Viện Nghiên cứu kinh tể phát triển thế giới, Trường Đại học Liên hợp cùng với Đại sứ quán Đan Mạch tại Việt Nam phối hợp thực hiện. Trước khi cuộc điều tra chính thức được tiến hành, các cuộc điều tra thử đã được thực hiện trước đó, cuộc điều tra được tiến hành thông qua các cuộc 
phỏng vấn sâu đối với hơn 2.552 DNNVV ngoài quốc doanh hoạt động trong khu vực chế biến, may mặc. Điều tra được thực hiện tại 10 tỉnh và thành phố bao gồm Hà Nội, Hải Phòng, thành phố Hồ Chí Minh, Hà Tây (cũ), Phú Thọ, Nghệ An, Quảng Nam, Khánh Hòa, Lâm Đồng và Long An.

Cách thức chọn mẫu năm 2015 tuân theo cách thức của các cuộc điều tra năm 2005, 2007, 2009 và 2011. Trên mọi lĩnh vực, các mẫu đều được phân tầng theo hình thức pháp lý để đảm bảo mọi loại hình doanh nghiệp ngoài quốc doanh đều được đưa vào bao gồm doanh nghiệp hộ gia đình, doanh nghiệp tư nhân, công ty hợp danh/hợp tác xã, công ty trách nhiệm hữu hạn và công ty cổ phần. Tổng số quan sát của bộ dữ liệu là 2.552 doanh nghiệp. Trong số doanh nghiệp này, có 266 DNNVV có thực hiện hợp đồng phụ được trích ra từ bộ dữ liệu điều tra DNNVV năm 2015. Đối tượng nghiên cứu của bài viết này là $\mathrm{DNNVV}$, nên trong quá trình sàn lọc thông tin từ dữ liệu, nghiên cứu đã loại bỏ 6 quan sát do thiếu thông tin. Do đó, tổng số quan sát của nghiên cứu này là 260 quan sát (chiếm 10,19\% tổng thể điều tra).

\section{2 Định nghĩa và đo lường các biến trong mô hình nghiên cứu}

Thông tin từ bộ dữ liệu điều tra DNNVV Việt Nam năm 2015 cho phép nghiên cứu này đo lường các biến trong mô hình nghiên cứu như sau:

Biến phụ thuộc (Y): HQHĐKD của doanh nghiệp là tỷ số giữa lợi nhuận trên tổng tài sản của doanh nghiệp (ROA). Biến này được tính bằng cách chia lợi nhuận ròng cho tổng tài sản của doanh nghiệp (câu hỏi kt3_13 và câu hỏi q73c).

\section{Các biến độc lập:}

Tỷ lệ nợ vay $\left(\mathrm{X}_{1}\right)$ : được phản ánh thông qua tỷ số của tổng nợ vay (câu hỏi q75) chia cho tổng tài sản (câu hỏi q73c).

Nguồn lực marketing $\left(\mathrm{X}_{2}\right)$ : được đo lường bởi tỷ số giữa chi phí dành cho hoạt động marketing (câu hỏi q57b_9) và tổng chi phí sản xuất gián tiếp (câu hỏi q57b_12).

Trình độ người quản lý doanh nghiệp $\left(\mathrm{X}_{3}\right)$ : được phản ảnh bởi biến giả, nhận giá trị 1 nếu người quản lý có bằng cấp chuyên môn từ trình độ cao đẳng và đại học trở lên và 0 cho các câu trả lời khác (câu hỏi q26c).

\section{Các biến kiểm soát:}

Quy mô doanh nghiệp $\left(\mathrm{X}_{4}\right)$ : được tính bằng cách giá trị logarit cơ số tự nhiên của tổng tài sản doanh nghiệp (câu hỏi q73c).

\section{Biến điều tiết:}

Hợp đồng phụ $(\mathrm{M})$ : được đo lường bằng tỷ số của doanh thu từ các hợp đồng phụ chia cho tổng doanh thu của doanh nghiệp (câu hỏi kt3_01).

\subsection{Phương pháp ước lượng}

Với phương pháp đo lường các biến ở trên, nghiên cứu này sử dụng mô hình hồi quy tuyến tính bằng phương pháp bình quân bé nhất (OLS) để ước lượng tác động của các nguồn lực đến HQHĐKD của doanh nghiệp cũng như vai trò điều tiết của yếu tố hợp đồng phụ trong các mối quan hệ đó. Phương trình ước lượng được thể hiện tổng quát thông qua phương trình (1).

\section{$\mathrm{Y}=\beta_{0}+\beta_{1} \mathrm{X}_{1}+\beta_{2} \mathrm{X}_{2}+\beta_{3} \mathrm{X}_{3}+\beta_{4} \mathrm{X}_{4}+\beta_{\mathrm{MX} 1} \mathrm{M}^{*} \mathrm{X}_{1}+\beta_{\mathrm{MX} 2} \mathrm{M}^{*} \mathrm{X}_{2}+\beta_{\mathrm{MX} 3} \mathrm{M}^{*} \mathrm{X}_{3+} \varepsilon$ (1)}

Trong đó, $\mathrm{Y}$ là biến phụ thuộc, $\beta_{0}$ là hệ số chặn của mô hình; $\beta_{1 \rightarrow 3}$ lần lượt là hệ số ước lượng các biến độc lập, $\beta_{4}$ là hệ số của yếu tố kiểm soát, $\beta_{\mathrm{MX1-3}}$ lần lượt là hệ số ước lượng các biến tương tác giữa biến điều tiết $\mathrm{M}$ với từng biến độc lập $\mathrm{X}$ tương ứng, $\mathrm{X}_{1->3}$ lần lượt là giá trị các biến độc lập, $\mathrm{X}_{4}$ là giá trị của yếu tố kiểm soát, $\mathrm{X}_{\mathrm{MX1->3}}$ lần lượt là giá trị của các biến tương tác giữa biến điều tiết $\mathrm{M}$ với từng biến độc lập $\mathrm{X}$ tương ứng, $\varepsilon$ là sai số của mô hình hồi quy.

\section{KẾT QUẢ VÀ THẢO LUẬN}

\subsection{Mô tả thống kê và ma trận tương quan}

Bảng 1 cho biết giá trị trung bình, độ lệch chuẩn, hệ số phóng đại phương sai (Variance inflation factor - VIF) và hệ số tương quan giữa các biến trong mô hình nghiên cứu. Kết quả phân tích ma trận tương quan ở Bảng 1 còn cho thấy hệ số tương quan giữa các biến độc lập đều thấp $(<0,8)$. Hệ số tương quan cao nhất 0,432 là mối tương quan giữa hai đại lượng thể hiện quy mô của nguồn vốn doanh nghiệp và trình độ của chủ doanh nghiệp. Bên cạnh đó, kết quả kiểm định cũng cho thấy tất cả các biến đều có 
giá trị VIF dưới ngưỡng giá trị 4,0. Các kết quả này hàm ý rằng, không xảy ra hiện tượng đa cộng tuyến (multicollinearity) khi xem xét tất cả các biến đồng thời trong một mô hình nghiên cứu [4].

Ngoài ra, kết quả kiểm định White cho biết rằng, dữ liệu của nghiên cứu không xảy ra hiện tượng phương sai sai số thay đổi (Heteroscedasticity) vì kiểm định này không có ý nghĩa về mặt thống kê với giá trị $\mathrm{p}=0,6549$. Các kết quả kiểm định trên ngầm mang ý nghĩa rằng, giá trị ước lượng các biến số trong mô hình nghiên cứu không bị chệch (unbiased estimation).

Bảng 1: Mô tả thống kê và ma trận tương quan giữa các biến trong mô hình (n=260)

\begin{tabular}{|c|c|c|c|c|c|c|c|c|}
\hline Các biến & $\begin{array}{l}\text { Trung } \\
\text { bình }\end{array}$ & $\begin{array}{l}\text { Độ lệch } \\
\text { chuẩn }\end{array}$ & VIF & 1 & 2 & 3 & 4 & 5 \\
\hline $\begin{array}{l}\text { 1. Hiệu quả } \\
\text { hoạt động kinh } \\
\text { doanh }\end{array}$ & 0,300 & 0,723 & & & & & & \\
\hline $\begin{array}{l}\text { 2. Quy mô } \\
\text { doanh nghiệp }\end{array}$ & 0,103 & 0,301 & 1,29 & $-0,156^{* *}$ & & & & \\
\hline 3. Tỷ lệ nợ vay & 0,013 & 0,041 & 1,07 & $0,555^{* * *}$ & $0,1544^{* *}$ & & & \\
\hline $\begin{array}{l}\text { 4. Nguồn lực } \\
\text { marketing }\end{array}$ & 0,331 & 0,471 & 1,12 & $-0,033$ & $0,240^{* * *}$ & $-0,023$ & & \\
\hline $\begin{array}{l}\text { 5. Trình độ chủ } \\
\text { doanh nghiệp }\end{array}$ & 14,556 & 1,692 & 1,35 & $0,112^{*}$ & $0,432^{* * *}$ & $0,208^{* * *}$ & $0,288^{* * *}$ & \\
\hline $\begin{array}{l}\text { 6. Thực hiện } \\
\text { hợp đồng phụ }\end{array}$ & 0,224 & 0,671 & 1,03 & $0,509^{* * *}$ & $-0,125$ & $-0,058$ & $-0,048$ & 0,044 \\
\hline
\end{tabular}

Ghi chú: *, **, *** là̀n luợt biểu diễn giá trị mức ý nghĩa thống kê tại 10\%, 5\% và 1\%

\subsection{Thảo luận kết quả}

Kết quả ước lượng mô hình hồi quy tuyến tính bằng phương pháp OLS về ảnh hưởng điều tiết của việc thực hiện hợp đồng phụ đến mối quan hệ giữa nguồn lực và HQHĐKD của DNNVV tại Việt Nam được trình bày trong Bảng 2 .

\section{Ảnh hưởng của nguồn lục doanh nghiệp đến HQHĐKD}

Đầu tiên, bài viết nghiên cứu ảnh hưởng của các nguồn lực doanh nghiệp đến HQHĐKD (mô hình 1 \& 2). Mô hình 1 chỉ xem xét tác động của yếu tố kiểm soát quy mô doanh nghiệp đến HQHĐKD của doanh nghiệp. Với hệ số $\mathrm{R}^{2}$ điều chỉnh, giá trị $\mathrm{P}$ của mô hình lần lượt là $0,025,0,012$ có nghĩa là sự thay đổi của các biến kiểm soát ở mô hình này giải thích được 2,5\% sự thay đổi trong HQHĐKD của doanh nghiệp với mức ý nghĩa thống kê tại $5 \%$. Kết quả từ mô hình 1 cho thấy rằng, tác động của quy mô doanh nghiệp có mối tương quan nghịch đến $\mathrm{HQHĐKD} \mathrm{của} \mathrm{DNNVV} \mathrm{tại} \mathrm{mức} \mathrm{ý} \mathrm{nghĩa} \mathrm{thống} \mathrm{kê} \mathrm{là} 5 \%$ ( $\beta=-$ $0,067 ; \mathrm{p}<0,05)$. Như vậy, yếu tố kiểm soát quy mô doanh nghiệp được tìm thấy có mối liên hệ đến HQHĐKD, tuy nhiên, mối liên hệ này lại là nghịch biến. Dù kết quả này khác với kết quả đã tìm thấy mối liên hệ đồng biến giữa quy mô doanh nghiệp với HQHĐKD trong các nghiên cứu trước [9], [11], nhưng lại đồng nhất với kết quả từ nghiên cứu của [16] và [13] khi kết luận yếu tố quy mô có tương quan nghịch với hiệu quả sản xuất. Theo [15], hiện tượng này có thể giải thích qua lý thuyết lựa chọn ngược: các DNNVV có xu hướng duy trì quy mô nhỏ để nhận được nhiều ưu đại từ chính phủ. Hơn nữa, trong bối cảnh kinh tế thế giới và trong nước có nhiều biến động bất ổn, khi quy mô gia tăng, DNNVV cũng phải đồng thời tăng cường khả năng quản lý, điều hành, phân phối đầu ra, v.v. Như vậy, khi việc mở rộng quy mô tồn tại những rủi ro lớn và trở thành gánh nặng, một số doanh nghiệp chọn cách giữ nguyên hoặc thu nhỏ quy mô cho phù hợp với khả năng điều hành và quản lý đã cho thấy tín hiệu tích cực và hiệu quả hơn trong kết quả hoạt động kinh doanh.

Từ nền tảng ở mô hình 1 , mô hình 2 cho biết kết quả ước lượng khả năng giải thích cho biến phụ thuộc khi thêm vào các biến độc lập và biến điều tiết. Giá trị $\mathrm{R}^{2}$ điều chỉnh của mô hình 2 là 0,640 , tương đương với khả năng giải thích lên đến $64 \%$ sự thay đổi của biến phụ thuộc, tăng đáng kể so với mô hình 1 $(2,5 \%)$. Điều này hàm ý rằng, tầm quan trọng của các biến độc lập thể hiện nguồn lực doanh nghiệp - tỷ lệ nợ vay, nguồn lực marketing, và trình độ chủ doanh nghiệp cần được xem xét trong mô hình. Bên cạnh 
đó, giá trị của p là 0,000 ; có nghĩa là mô hình 2 có ý nghĩa thống kê ở mức $1 \%$. Kết quả ước lượng từ mô hình 2 cho thây, khi xem xét như một biến độc lập bình thường, việc thực hiện hợp đồng phu có mối tương quan thuận với $H Q H Đ K D$ tại mức ý nghĩa thông kê là $1 \%(\beta=0,559 ; \mathrm{p}<0,001)$.

Bảng 2: Kết quả mô hình hồi quy tuyến tính (OLS) nguồn lực ảnh hưởng đến hiệu quả HĐKD của DNNVV $(\mathrm{N}=260)$

\begin{tabular}{|c|c|c|c|c|c|c|c|}
\hline Các biến & $\begin{array}{c}\text { Ký } \\
\text { hiệu }\end{array}$ & Mô hình 1 & Mô hình 2 & Mô hình 3 & Mô hình 4 & Mô hình 5 & Mô hình 6 \\
\hline Hằng số & & $1,274 * * *$ & $1,330 * * *$ & $1,358 * * *$ & $1,356 * * *$ & $1,510 * * *$ & $1,533 * * *$ \\
\hline \multicolumn{8}{|l|}{ Biến độc lập } \\
\hline Tỷ lệ nợ & $\mathbf{X}_{1}$ & & $1,466 * * *$ & $1,718 * * *$ & $1,460 * * *$ & $1,470 * * *$ & $1,470 * * *$ \\
\hline Nguồn lực marketing & $\mathbf{X}_{2}$ & & 0,788 & 0,870 & $1,651 *$ & 0,750 & $1,548 * *$ \\
\hline $\begin{array}{l}\text { Trình độ chủ doanh } \\
\text { nghiệp }\end{array}$ & $\mathbf{X}_{3}$ & & 0,065 & 0,044 & 0,080 & $-0,091$ & $-0,076$ \\
\hline \multicolumn{8}{|l|}{ Biến kiểm soát } \\
\hline Quy mô doanh nghiệp & $\mathbf{X}_{4}$ & $-0,067 * *$ & $-0,092 * * *$ & $-0,094 * * *$ & $-0,094 * * *$ & $-0,095 * * *$ & $-0,097 * * *$ \\
\hline \multicolumn{8}{|l|}{ Biến điều tiết } \\
\hline Hợp đồng phụ (HĐP) & $\mathbf{M}$ & & $0,559 * * *$ & $0,578 * * *$ & $0,567 * * *$ & $-0,090$ & $-0,077$ \\
\hline \multicolumn{8}{|l|}{ Biến tương tác } \\
\hline HĐP x Tỷ lệ nợ & $\mathbf{M X}_{1}$ & & & $-2,590 * *$ & & & $-0,058$ \\
\hline $\begin{array}{l}\text { HĐP x Nguồn lực } \\
\text { marketing }\end{array}$ & $\mathbf{M X}_{2}$ & & & & $-9,372^{*}$ & & $-8,651^{*}$ \\
\hline HĐP x Trình độ chủ DN & $\mathbf{M X}_{3}$ & & & & & $0,760 * * *$ & $0,754 * * *$ \\
\hline Prob $>$ F & & 0,012 & 0,000 & 0,000 & 0,000 & 0,000 & 0,000 \\
\hline $\mathbf{R}^{2}$ điều chỉnh & & 0,025 & 0,640 & 0,648 & 0,645 & 0,703 & 0,707 \\
\hline$\Delta \mathbf{R}^{2}$ & & & & 0,008 & 0,005 & 0,063 & 0,067 \\
\hline F change & & & & $5,99 * *$ & $3,40 *$ & $52,98 * * *$ & $18,93 * * *$ \\
\hline
\end{tabular}

Ghi chú: *,**, *** là̀n luợt biểu diến giá trị múc ý nghĩa thống kê tại 10\%, 5\% và 1\%; MH: Mô hình

Điều này có nghĩa là tỷ lệ doanh thu từ các hợp đồng phụ trên tổng doanh thu càng tăng thì sẽ làm tăng HQHĐKD. Tương tự, biến tỷ lệ nợ vay cũng cho thấy mối tương quan thuận đến biến phụ thuộc tại mức ý nghĩa $1 \%(\beta=1,466 ; p<0,001)$. Kết quả này hoàn toàn ủng hộ giả thuyết 1 đã được đặt ra trong mô hình, đó là tỷ lệ nợ của doanh nghiệp có mối quan hệ đồng biến với HQHĐKD của doanh nghiệp.

Sau đó, các biến tương tác tương ứng với biến điều tiết và từng biến độc lập được lần lượt xem xét lần lượt, kết quả được trình bày tương ứng tại các mô hình $3,4,5$ và 6 . Từ Bảng 2 , ta thấy giá trị $\mathrm{R}^{2}$ có sự cải thiện đáng kể so với giá trị $\mathrm{R}^{2}$ điều chỉnh ở các mô hình 1 và 2 .

Trước tiên, khi đưa thêm biến tương tác giữa hợp đồng phụ và tỷ lệ nợ $\left(\mathrm{MX}_{1}\right)$ vào mô hình 3 , khả năng giải thích đến biến phụ thuộc hiện tại đã đạt $64,8 \%$ tại mức ý nghĩa $1 \%(\mathrm{p}=0,000)$, cải thiện không đáng kể so với giá trị $\mathrm{R}^{2}$ điều chỉnh ở mô hình $2(64 \%)$. Dầu vậy, giá trị thay đồi của $\mathrm{R}^{2}$ điều chỉnh từ mô hình 2 sang mô hình 3 được ước lượng có ý nghĩa thống kê ở mức $5 \%\left(\Delta \mathrm{R}^{2}=0,008, \mathrm{~F}(1,253)=5,99\right.$, $\mathrm{p}<0,05)$. Kết quả này chứng tỏ rằng khả năng giải thích của tỷ lệ nợ vay dến $H Q H Đ K D$ chịu sự điều tiết tuyến tính của việc thực hiện hợp đồng phụ. Bên cạnh đó, mô hình 3 đã cho thấy được kết quả ước lượng của biến tương tác giữa hợp đồng phụ và tỷ lệ nợ vay tại mức ý nghĩa thống kê $5 \%(\beta=-2,590 ; p<0,05)$. Với kết quả ước lượng này ta thấy, hệ số của biến $\mathrm{MX}_{1}$ mang dấu âm, hàm ý rằng mức độ thực hiện hợp 
đồng phụ đang có tác động kiềm hãm lên mối quan hệ giữa tỷ lệ nơ vay và $H Q H Đ K D$. Kết quả này khẳng định giả thuyết $4 \mathrm{a}$ được ủng hộ cả về lý thuyết lẫn thực tiễn. Ngoài ra, đối vối các biến độc lập và kiểm soát được kế thừa từ mô hình 2 , kết quả ở mô hình 3 không có sự khác biệt cả về dấu ảnh hưởng và ý nghĩa thống kê.

Kế tiếp, quan sát kết quả ước lượng từ mô hình 4 ta thấy được, giá trị $\mathrm{R}^{2}$ điều chỉnh của mô hình đạt 0,645 , tương đương với khả năng giải thích sự biến động của biến phụ thuộc đến $64,5 \%$, và mô hình đạt mức ý nghĩa thống kê $1 \%$. Thêm vào đó, khi đưa thêm biến tương tác giữa hợp đồng phụ và nguồn lực marketing $\left(\mathrm{MX}_{2}\right)$, giá trị ước lượng $\Delta \mathrm{R}^{2}$ điều chỉnh ở mô hình 4 so với mô hình 2 cho thấy có ý nghĩa thống kê ở mức $10 \%\left(\Delta \mathrm{R}^{2}=0,005, \mathrm{~F}(1,253)=3,40, \mathrm{p}<0,1\right)$. Tóm lại, tuy mô hình 4 đạt độ phù hợp kém hơn mô hình 3 , nhưng kết quả ước lượng của mô hình vẫn có giá trị khẳng định sự tồn tại của ảnh hưởng điều tiết từ hợp đồng phụ đến mối quan hệ nguồn lực marketing - HQHĐKD. Bên cạnh đó, xét sự thay đổi của tất cả các biến trong mô hình 4 , khá tương tự như mô hình 3 , các biến kiểm soát, điều tiết và đa số các biến độc lập đều không cho thấy sự thay đổi về dấu tương quan hay mức ý nghĩa. Tuy nhiên, đặc biệt ở trường hợp đưa thêm vào mô hình biến tương tác giữa nguồn lục marketing và hợp đồng $p h u\left(\mathrm{MX}_{2}\right)$, hệ số của biến độc lập về nguồn lực marketing $\left(\mathrm{X}_{2}\right)$ từ kết quả không có ý nghĩa thống kê ở mô hình 2 và 3 , đã cho thấy có ý nghĩa thống kê ở mức $10 \%$ ở mô hình $4(\beta=1,651 ; \mathrm{p}<0,1)$. Điều này mang hàm ý rằng, so với các mô hình trước thì biến nguồn lực marketing phù hợp hơn với mô hình 4 - mô hình có sự xuất hiện của biến tương tác giữa nguồn lực marketing và hợp đồng phụ. Một kết quả ước lượng quan trọng khác từ mô hình 4 đó là hệ số của biến tương tác có ý nghĩa thống kê ở mức $10 \%(\beta=-9,372 ; \mathrm{p}<0,1)$. Kết quả này mang hàm ý tương tự mô hình 3 đó là, mức độ thực hiện hợp đồng phụ đang có tác động kiềm hãm lên mối quan hệ giữa nguồn lực marketing và $H Q H Đ K D$. Do đặc thù của loại hình kinh doanh này là các nhà thầu phụ quy mô nhỏ chịu lệ thuộc vào các đối tác lớn, họ phải sản xuất theo các thông số kỹ thuật cũng như rất nhiều yêu cầu chi tiết khác được thể hiện trong hợp đồng. Từ đó, các DNNVV này phải điều chỉnh chiến lược marketing của mình cho phù hợp với từng đối tượng khách hàng. Như vậy, giả thuyết $4 \mathrm{~b}$ được ủng hộ về mặt lý luận lẫn thực tiễn.

Sau đó, tác giả xét đến mô hình 5 , bằng việc bổ sung của biến tương tác giữa hợp đồng phụ và trình độ chủ doanh nghiệp, $\mathrm{R}^{2}$ đạt giá trị $70,3 \%$ và mô hình đạt mức ý nghĩa thống kê $1 \%(\mathrm{p}=0,000)$. Ngoài ra, giá trị thay đổi của $\mathrm{R}^{2}$ điều chỉnh từ mô hình 2 sang mô hình 5 được ước lượng có ý nghĩa thống kê ở mức $1 \%\left(\Delta \mathrm{R}^{2}=0,063, \mathrm{~F}(1,253)=52,98, \mathrm{p}<0,001\right)$. Như vậy, các kết quả trên đều chứng tỏ được mô hình 5 phù hợp hơn về khả năng giải thích của biến tương tác $\left(\mathrm{MX}_{3}\right)$ lên mối quan hệ giữa biến độc lập tương ứng $\left(\mathrm{X}_{3}\right)$ với biến phụ thuộc tốt hơn so với các biến tương tác trước ở mô hình 3 và 4 . Một điểm nổi bật khác có thể nhận xét từ kết quả ước lượng của mô hình 5 đó là biến tương tác có hệ số tương quan và mức ý nghĩa tốt hơn hẳn các biến tương tác ở mô hình 3 và 4 , cụ thể là $\beta=0,760 ; p<0,001$. Kết quả này mang hàm ý rằng, ảnh hưởng điều tiết của hợp đồng phụ tác động mạnh mẽ đến tác nhân con người hơn hai tác nhân là tỷ lệ nợ và nguồn lực marketing. Thật vậy, theo [8], với những kiến thức và kinh nghiệm chuyên môn, nếu người quản lý doanh nghiệp có thể tiếp cận với một mạng lưới kinh doanh hiệu quả với cộng đồng các doanh nghiệp cùng ngành cũng như các đối tác tiềm năng khác thông qua việc thực hiện hợp đồng phụ. Tóm lại, kết quả thực tiễn này đã phù hợp với kỳ vọng của giả thuyết $4 \mathrm{c}$.

Mô hình 6 là mô hình tổng hợp của nghiên cứu này, bao gồm tất cả các biến kiểm soát, độc lập, điều tiết cùng các biến tương tác tương ứng được ước lượng đồng thời trong cùng một mô hình. Kết quả mô hình 6 cho thấy, $\mathrm{R}^{2}$ điều chỉnh là cao nhất với giá trị là $70,3 \%$, và mô hình đạt mức ý nghĩa thống kê $1 \%$ $(\mathrm{p}=0,000)$. Điều này cho thấy rằng, kết quả ước lượng ở mô hình 6 dùng để giải thích sự thay đổi của biến kiểm soát, các biến độc lập, điều tiết và tương tác đến sự biến động của biến phụ thuộc là tốt nhất. Hơn nữa, giá trị thay đổi của $\mathrm{R}^{2}$ điều chỉnh ở mô hình 6 này so với mô hình 2 được ước lượng có ý nghĩa thống kê cao ở mức $1 \%\left(\Delta \mathrm{R}^{2}=0,067, \mathrm{~F}(1,253)=18,93, \mathrm{p}<0,001\right)$. Kết quả này một lần nữa chứng minh khả năng giải thích của các nguồn lực doanh nghiệp đến $H Q H Đ K D$ chịu sự điều tiết tuyến tính của việc thực hiện hợp đồng phụ. Cụ thể, trong số 3 biến tương tác ở mô hình 6 , trừ biến tương tác giữa tỷ lệ nợ và hợp đồng phụ là không có ý nghĩa thống kê, 2 biến tương tác có ý nghĩa thống kê còn lại cho kết quả ước lượng hầu như không khác với kết quả khi quan sát độc lập từng biển tương tác, cả về dấu tương quan và mức ý nghĩa (hệ số ước lượng cho $\mathrm{MX}_{2}$ và $\mathrm{MX}_{3}$ lần lượt là $\beta=-8,651 ; \mathrm{p}<0,1$ và $\beta=0,754 ; \mathrm{p}<0,001$ ). Tuy nhiên, độ lớn của hệ số tương quan ứng với các biến tương tác $\mathrm{MX}_{2}$ và $\mathrm{MX}_{3}$ đều giảm so với khi các biến này được quan sát riêng lẻ trước đó lần lượt ở mô hình 4 và 5 . Điều này mang hàm ý rằng, với các 
DNNVV thực hiện hợp đồng phụ, khi chủ doanh nghiệp có trình độ học vấn càng cao thì càng có giải pháp để quản lý nguồn lực marketing hiệu quả hơn và không để nợ xảy ra. Cuối cùng, đối với biến kiểm soát và các biến độc lập còn lại, kết quả ở mô hình 6 không có sự khác biệt so với các mô hình 1, 2, 3, 4 và 5 về dấu ảnh hưởng cũng như mức ý nghĩa thống kê.

\section{KẾT LUẬN}

Lựa chọn phân bổ nguồn lực có hạn của các DNNVV một cách hợp lý là một trong những chiến lược quan trọng nhằm đạt được kết quả hoạt động kinh doanh hiệu quả. Bằng cách tiếp cận theo Lý thuyết nguồn lực, bài viêt đã phát triển những tranh luận về mối quan hệ giữa nguồn lực doanh nghiệp và HQHĐKD. Bằng chứng thực tiễn từ 260 DNNVV có thực hiện hợp đồng phụ tại Việt Nam cho thấy rằng, tỷ lệ nợ vay của doanh nghiệp có quan hệ đồng biến với HQHĐKD. Ngoài ra, kết quả nghiên cứu còn cho thấy rằng với vai trò là biến điều tiết, mức độ thực hiện hợp đồng phụ đã tác động đáng kể đến mối quan hệ của nguồn lực doanh nghiệp và HQHĐKD. Cụ thể, ảnh hưởng đồng biến của tỷ lệ nợ vay và nguồn lực marketing đến $\mathrm{HQHĐKD} \mathrm{của} \mathrm{doanh} \mathrm{nghiệp} \mathrm{là} \mathrm{yếu} \mathrm{hơn} \mathrm{hơn} \mathrm{khi} \mathrm{doanh} \mathrm{nghiệp} \mathrm{tăng} \mathrm{thực} \mathrm{hiện} \mathrm{hợp} \mathrm{đồng}$ phụ. Trong khi đó, trình độ chủ doanh nghiệp có ảnh hưởng mạnh hơn đến HQHĐKD khi doanh nghiệp tăng thực hiện hợp đồng phụ. Hàm ý của nghiên cứu này là các nhà thầu phụ quy mô nhỏ và vừa tại Việt Nam có thể lựa cách tăng cường thực hiện hợp đồng phụ nhằm khai thác hiệu quả hơn nguồn lực về trình độ chủ doanh nghiệp. Ngoài ra, các chính sách hỗ trợ cho đối tượng các doanh nghiệp này cũng nên tập trung hơn về mặt vay vốn và các khía cạnh marketing (chiêu thị, phân phối, v.v.).

Cụ thể hơn, hàm ý rút ra từ kết quả nghiên cứu về tác động điều tiết của hợp đồng phụ là: (1) hiệu quả của việc sử dụng đòn bẫy tài chính nhằm nâng cao HQHĐKD là rất cao, tuy nhiên, các DNNVV muốn thực hiện hợp đồng phụ ở Việt Nam thì không nên sử dụng chiến lược này; (2) các DNNVV càng tăng cường nguồn lực cho marketing thì $H Q H Đ K D$ của các doanh nghiệp này càng tăng nhưng mối liên hệ này sẽ yếu đi khi doanh nghiệp càng thực hiện nhiều hợp đồng phụ do không làm chủ được chuỗi giá trị và chiến lược quản trị cho nguyên liệu đầu vào đến thành phẩm cuối cùng; (3) DNNVV càng tăng thực hiện hợp đồng phụ thì trình độ chủ doanh nghiệp càng được sử dụng trong hoạt động kinh doanh hiệu quả do có thể tận dụng các mối quan hệ hợp tác để tiếp cận được lợi ích bổ sung như thông tin về thị trường hay kinh nghiệm và những bài học về quản trị tri thức trong doanh nghiệp.

Ngoài ra, kết quả ước lượng về quy mô doanh nghiệp cho thấy rằng người quản lý nên am hiểu tường tận về doanh nghiệp của mình nhằm có được những đánh giá, chiến lược thay đổi quy mô phù hợp nhất với mô hình kinh doanh của doanh nghiệp mình.

Bài viết này nghiên cứu dưới góc nhìn của các nhà thầu phụ công nghiệp - phía cung ứng trong nền công nghiệp hỗ trợ. Do vậy, các nghiên cứu tiếp theo trong tương lai có thể xem xét dưới góc nhìn từ bên ngược lại - phía cầu của nền công nghiệp hỗ trợ để có được cái nhìn toàn diện và khách quan. Ngoài ra, đối tượng nghiên cứu trong bài viết này là các DNNVV hoạt động trong lĩnh vực chế tạo, lắp ráp nên các nghiên cứu kế tiếp về HQHĐKD trong doanh nghiệp nên tiến hành trên các doanh nghiệp với lĩnh vực hoạt động đa dạng hơn cũng như nghiên cứu cả các yếu tố bên ngoài doanh nghiệp.

\section{LÒ̀I CẢM ƠN}

Tác giả chân thành cảm ơn Quỹ Phát triển khoa học và công nghệ Quốc gia (NAFOSTED) trong đề tài mã số 502.02-2015.13 đã tài trợ cho nghiên cứu này.

\section{TÀI LIỆU THAM KHẢO}

[1] Barney, J. (1991), "Firm resources and sustained competitive advantage", Journal of management, 17(1), 99120.

[2] Camuffo, A., Furlan, A., Romano, P., \& Vinelli, A. (2007), "Routes towards supplier and production network internationalization", International Journal of Operations and Production Management, 27(4), 371-387.

[3] Hansen, H., Rand, J. \& Tarp, F. (2004), "SME Growth and Survival in Vietnam: Did Direct Government Support Matter?”, www.tandfonline.com/doi/full/10.1080/00220380902811025, đăng nhập ngày 20/12/2016. 
[4] Hair, J. F., Black, W. C., Babin, B. J., Anderson, R. E., \& Tatham, R. L. (2006). Multivariate Data Analysis, Pearson Prentice Hall, Upper Saddle River, NJ.

[5] Hayashi, M. (2002), "The role of subcontracting in SME development in Indonesia: Micro-level evidence from the metalworking and machinery industry”, Journal of Asian Economics, 13(1), 1-26.

[6] Jensen, M. C. (1986), "Agency cost of free cash flow, corporate finance, and takeovers, Corporate Finance, and Takeovers", American Economic Review, 76(2), 323-329.

[7] Kawasaki, S. \& McMillan J. (1987), "The Design of Contracts: Evidence from Japanese Subcontracting”, Journal of the Japanese and International Economies, 1(3), 327-349.

[8] Kim, J., \& Hemmert, M. (2016), "What drives the export performance of small and medium-sized subcontracting firms? A study of Korean manufacturers”, International Business Review, 25(2), 511-521.

[9] Mai Văn Nam \& Nguyễn Quốc Nghi (2011), "Các nhân tố ảnh hưởng đến hiệu quả hoạt động kinh doanh của Doanh nghiệp nhỏ và vừa ở Thành phố Cần Thơ", Tạp chí Khoa học Triờng Đại Học Cần Tho; 19b, 122-129.

[10] Majumdar, S.K. (1997), "The impact of size and age on firm-level performance: Some evidence from India", Review of Industrial Organization, 12(2), 231-241.

[11] Martani, D., \& Khairurizka, R. (2009), "The effect of financial ratios, firm size, and cash flow from operating activities in the interim report to the stock return", Chinese Business Review, 8(6), 44-55.

[12] Nickell, S., \& Nicolitsas, D. (1999), "How does financial pressure affect firms?”, European Economic Review, 43(8), 1435-1456.

[13] Nikaido, Y. (2004), "Technical efficiency of small-scale industry: application of stochastic production frontier model", Economic and Political Weekly, 592-597.

[14] Quan Minh Nhựt (2010), “Các nhân tố tác động đến hiệu quả sản xuất của các doanh nghiệp chế biến thủy sản khu vực Đồng bằng sông Cửu Long”, Tạp chí Khoa học Truoòng Đại học Cần Tho, 13, 137-143.

[15] Trương Đông Lộc \& Nguyễn Đức Trọng (2010), "Hiệu quả sản xuất kinh doanh của các DNVVN Đồng bằng sông Cửu Long", Tạp chí Công nghệ ngân hàng, 50, 11-16.

[16] Võ Hồng Đức \& Lê Hoàng Long (2014), "Mạng lưới kinh doanh và hiệu quả sản xuất của doanh nghiệp nhỏ và vừa: Bằng chứng thực nghiệm từ ngành sản xuất thực phẩm và đồ uống”, Tạp chí Phát triển kinh tế, 286, 50-69.

[17] Võ Xuân Vinh \& Đoàn Thị Lệ Chi (2014), "Dòng tiền tự do và hiệu quả hoạt động của các doanh nghiệp Việt Nam”, Tạp chí Phát triển Kinh tế, 280, 61-77.

[18] Watson, J. (2007), "Modeling the relationship between networking and firm performance", Journal of Business Venturing, 22, 852-874.

[19] Wernerfelt, B. A. (1984), "Resource-based view of the firm", Strategic Management Journal, 5, 171-180.

Ngày nhận bài: 27/04/2017

Ngày chấp nhận đăng: 10/10/2017 